\title{
Anisotropic Magnetic Resonance in Random Nanocrystal Quantum Dot Ensembles
}

\author{
António J. S. Almeida, Ayaskanta Sahu, David J. Norris, Gleb N. Kakazei, Haripriya Kannan, \\ Martin S. Brandt, Martin Stutzmann, and Rui N. Pereira*
}

Cite This: ACS Omega 2020, 5, 11333-11341

Read Online

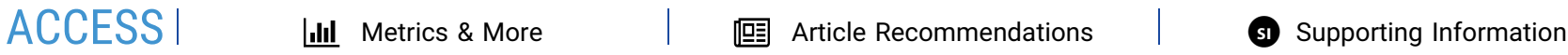

ABSTRACT: Magnetic anisotropy critically determines the utility of magnetic nanocrystals (NCs) in new nanomagnetism technologies. Using angular-dependent electron magnetic resonance (EMR), we observe magnetic anisotropy in isotropically arranged NCs of a nonmagnetic material. We show that the shape of the EMR angular variation can be well described by a simple model that considers magnetic dipole-dipole interactions between dipoles randomly located in the NCs, most likely due to surface dangling bonds. The magnetic anisotropy results from the fact that the energy term arising from the magnetic dipole-dipole
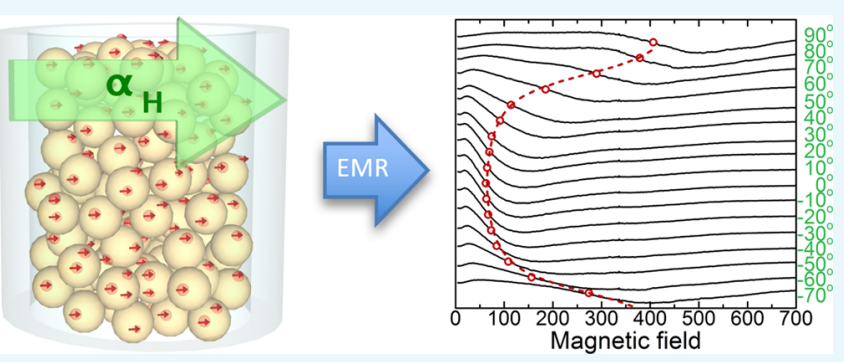
interactions between all magnetic moments in the system is dominated by only a few dipole pairs, which always have an anisotropic geometric arrangement. Our work shows that magnetic anisotropy may be a general feature of NC systems containing randomly distributed magnetic dipoles.

\section{INTRODUCTION}

Nanocrystals (NCs) of inorganic materials display a wealth of remarkable properties that arise from their reduced dimensions. ${ }^{1-7}$ In particular, ensembles of magnetic NCs have been the subject of intense research ${ }^{8-16}$ due to their potential in applications such as high-density recording media, $9,13,17,18$ spintronics, ${ }^{19,20}$ magnetic resonance imaging, ${ }^{21,22}$ and heat induction by the magnetocaloric effect ${ }^{23,24}$ for use in cancer therapy, $^{22,23}$ drug delivery, ${ }^{25,26}$ treatment of diseases by hyperthermia, ${ }^{27}$ thermal imaging, ${ }^{28}$ and remote control of ion channels in cells. ${ }^{29}$

Many of these applications rely on magnetic anisotropy to preserve the orientation of the magnetization against for example thermal fluctuations., $17-21,23$ Magnetic anisotropy may occur in an ensemble of NCs when each NC is magnetically anisotropic, for example, due to magnetocrystalline anisotropy, ${ }^{9,18,23}$ due to an anisotropic NC shape, ${ }^{17,20,21}$ or due to strain, ${ }^{19}$ and the NCs are arranged such that their magnetization easy axes are parallel..$^{9,17,18}$ For example, NCs with magnetocrystalline anisotropy ordered in arrays ${ }^{9}$ or in superlattices formed by thermal annealing ${ }^{18}$ have been shown to support stable magnetization reversal transitions and to provide stable elementary bits for nanorecording. Highly tunable dielectric properties with weak magnetic fields have also been achieved by embedding magnetically polarized $\mathrm{Ni}$ $\mathrm{NCs}$ in a diamagnetic $\mathrm{BaTiO}_{3}$ matrix. ${ }^{30} \mathrm{NCs}$ have proven valuable in enhancing the properties of host materials when embedded in other material matrices. ${ }^{30-33}$ Ensembles of randomly oriented NCs may also show magnetic anisotropy if the NCs are stacked in a volume with anisotropic shape, such as a thin film, ${ }^{34-36}$ a chain, ${ }^{20,37-39}$ or an elongated elipsoid. ${ }^{40}$ This magnetic anisotropy has been described within the framework of uniformly finite magnetic bodies with an effective demagnetization tensor having the same symmetry as the NC sample shape, ${ }^{34,36,37,41}$ in a way similar to that applied for bulk magnetic materials. ${ }^{42}$ Some studies considered magnetic dipole-dipole interactions explicitly to link the symmetry of the demagnetization tensor to the shape of the NC ensemble. ${ }^{35}$ Magnetic dipole-dipole interactions have also been proposed to be the origin of other new magnetic phenomena discovered in NC systems, such as collective interactions in self-assembled binary NC superlattices leading to a single-phase-like magnetization alignment that is different from the behavior of phaseseparated mixtures of the two types of $\mathrm{NCs}^{16}$ or the appearance of correlated areas of parallel magnetization in two-dimensional self-assemblies of superparamagnetic Co NCs. ${ }^{15}$ Moreover, it has been predicted that NCs of diamagnetic materials can exhibit magnetic properties, in particular that $\mathrm{CdSe}$ NCs should possess macroscopic magnetic moments resulting from dangling bond magnetic polarons. ${ }^{43}$ This prediction has been supported recently by the

Received: January 21, 2020

Accepted: April 15, 2020

Published: May 13, 2020 
experimental observation of magnetic polarons in $\mathrm{CdSe}$ colloidal NCs in fluorescence line narrowing (FLN) experiments. $^{44}$

In this work, we report the observation of magnetic anisotropy in ensembles of CdSe NCs randomly stacked in an isotropic shape and show that this unexpected behavior can be explained by considering magnetic dipole-dipole interactions between dipoles in the NCs. The magnetic anisotropy is probed via the angular dependence of electron magnetic resonance (EMR) spectra and results from the fact that the energy term arising from the magnetic dipole-dipole interactions between all magnetic moments in the system is dominated by only a few dipole pairs, which always have an anisotropic geometric arrangement. Our study provides insight on the role of magnetic dipole-dipole interactions on the magnetic behavior of nanomaterials by showing that these interactions may yield magnetic anisotropy even in NC ensembles lacking shape anisotropy and magnetic atoms.

\section{RESULTS AND DISCUSSION}

Figure 1 shows the EMR spectra recorded at room temperature for two samples of (undoped) CdSe NCs,

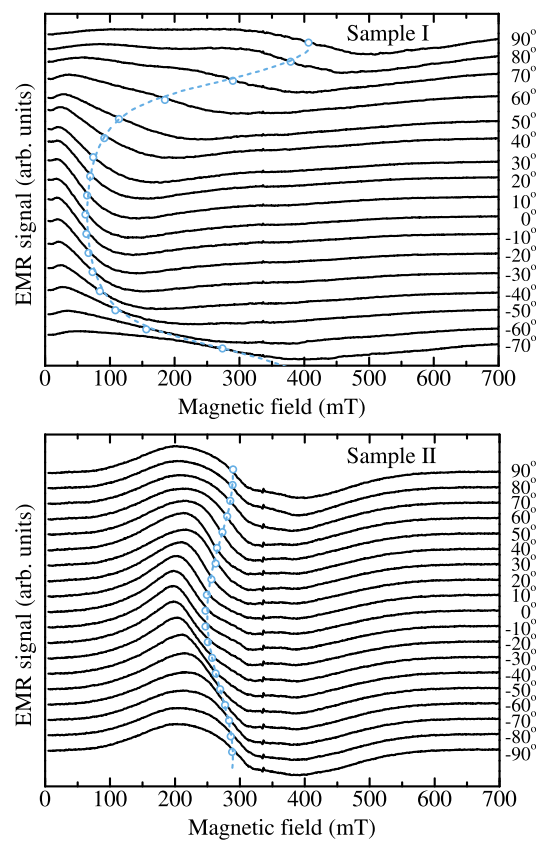

Figure 1. EMR spectra recorded for two CdSe NC samples with $\mathbf{H}$ at different angles, $\alpha_{\mathrm{H}}$ within the $x y$-plane. The open dots indicate the zero-crossing magnetic field, $H_{\text {res }}$ of the resonance band observed in each spectrum. The dashed lines show the $H_{\text {res }}$ angular variation calculated with the model described in the text.

prepared from the same synthesis batch, for various orientations of the external magnetic field, $\mathbf{H}$. Here, $\mathbf{H}$ was rotated in the $x y$-plane, which is perpendicular to the sample tube axis ( $z$-axis); see Figure 3 a. As can be seen, each EMR spectrum displays a resonance band with a zero-crossing magnetic field, $H_{\text {res }}$ that depends on the $\mathbf{H}$ orientation, $\alpha_{\mathrm{H}}$. The location of $H_{\text {res }}$ in each spectrum is indicated by the dots in Figure 1. For simplicity, $\alpha_{\mathrm{H}}$ is set with respect to the $\mathbf{H}$ orientation for which the resonance band displays the lowest $H_{\text {res. }}$. For sample I, the lowest and highest $H_{\text {res }}$ are located at 62 $\mathrm{mT}\left(\alpha_{\mathrm{H}}=0^{\circ}\right)$ and $\sim 400 \mathrm{mT}\left(\alpha_{\mathrm{H}}=90^{\circ}\right)$, respectively. A qualitatively similar angular dependence is observed for sample II, but with a considerably smaller amplitude of variation in $H_{\text {res. }}$ Sample II also shows a sharp signal without angulardependent shape and position which is located at $\approx 335 \mathrm{mT}$ and is related to paramagnetic Se vacancies in the NCs. ${ }^{45} \mathrm{We}$ have measured 30 samples of CdSe NCs from six independent synthesis batches, with each batch comprising both undoped and Ag-doped samples. We observed angular-dependent EMR for all samples. However, the amplitudes of the $H_{\text {res }}$ angular dependence varied quite broadly from sample to sample, with observed amplitudes ranging from a few $\mathrm{mT}$ to $\sim 200 \mathrm{mT}$. No correlation between the amplitude of the EMR angular dependence and the synthesis batch or the Ag doping level was observed. This will be discussed further below, in the context of the results presented in Figure 5.

To display magnetic resonance, our NCs must contain magnetic dipoles. Recently, both room-temperature ferromagnetism $^{46-49}$ and paramagnetism ${ }^{50}$ have been reported for $\mathrm{CdSe}$ NCs surface-coated with trioctylphosphine oxide (TOPO) ligands. In fact, ferromagnetism has been recently reported for several types of organic-ligand-capped NCs made of materials which, like CdSe, are diamagnetic in the bulk such as $\mathrm{Au}^{51-56}$ and $\mathrm{ZnO} .^{57-59}$ In these studies, it was concluded that the magnetic behavior originates from magnetic dipoles located at the NC surface. Theoretical studies of CdSe NCs have predicted that the interaction between spins of surface dangling bonds may lead to the formation of magnetic polarons, making each NC ferromagnetic. ${ }^{43}$ Very recently, the formation of these dangling bond magnetic polarons in $\mathrm{CdSe}$ NCs has been demonstrated experimentally by observing via FLN spectroscopy a strong decrease in the radiative decay of the dark exciton at temperatures below the critical temperature of polaron formation. ${ }^{44}$

In our samples, the NC powder was randomly compacted into a volume without shape anisotropy when $\mathbf{H}$ is rotated within the $x y$-plane perpendicular to the sample-tube axis. For such a system, paramagnetic centers (noninteracting magnetic dipoles) can only induce isotropic lines in the magnetic resonance spectra, even if each paramagnetic center is locally anisotropic, for example, if each paramagnetic center lies at a crystal lattice site. ${ }^{60}$ This is because in a powder system, the symmetry axes of the different paramagnetic centers are randomly oriented with respect to the applied magnetic field and, therefore, the energy of the system loses any dependence on the magnetic field orientation. ${ }^{60}$ However, as shall be demonstrated below, for a system of interacting magnetic dipoles with mutually aligned magnetic moments, the energy of the system can be anisotropic as a result of the dipoledipole interaction, which is intrinsically anisotropic. The strength of the dipole-dipole interaction when the (mutually aligned) magnetic moments of the dipoles are parallel to the line connecting the dipoles is different from that when the magnetic moments are perpendicular to that line. Thus, the observed angular dependence in EMR spectra is a direct indication of the presence of interacting magnetic dipoles, via dipole-dipole interaction, in our NC ensembles. Presence of the aligned magnetic moments, that is, magnetic ordering, in our NC ensembles was confirmed by means of magnetization loop measurements.

Figure 2 shows representative magnetization loops measured for four CdSe NC samples prepared from different synthesis batches. The magnetization curves of our NCs display a saturation at values, $M_{\mathrm{s}}=2-6 \mathrm{memu} / \mathrm{g}$ and coercivity, $H_{\mathrm{c}}$ 


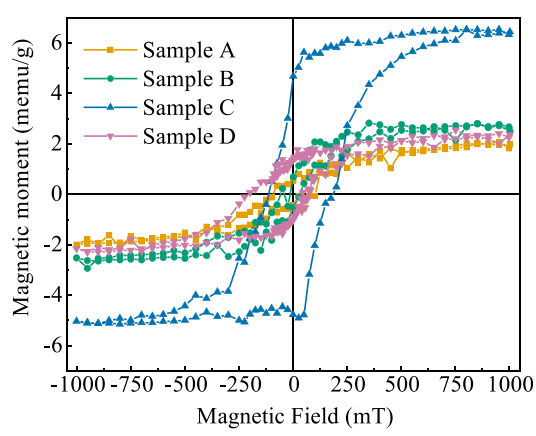

Figure 2. Magnetization loops measured with SQUID at room temperature for four of the CdSe NC samples analyzed in our study.

ranging from 20 to $200 \mathrm{mT}$. No trend is observed in the relation between $M_{\mathrm{s}}$ and $H_{\mathrm{c}}$. We can rule out that transitionmetal contaminants such as $\mathrm{Fe}$ are the origin of the magnetism of our CdSe NCs. If Fe $\left(M_{s}=14000 \mathrm{emu} / \mathrm{g}\right), \mathrm{Ni}\left(M_{\mathrm{s}}=4000\right.$ $\mathrm{emu} / \mathrm{g})$, and Co $\left(M_{\mathrm{s}}=12600 \mathrm{emu} / \mathrm{g}\right)$ in quantities of about 1 $\mathrm{ppb}$ were the source for the observed magnetization, our samples should display $M_{\mathrm{s}} \approx 0.02 \mathrm{memu} / \mathrm{g}$. This value is at least 2 orders of magnitude smaller than the values of $M_{s}$ measured in our samples. From magnetization loop measurements, we estimate an average number of magnetic dipoles per $\mathrm{NC}$ in the range of $10-30$, corresponding to the saturation magnetization times the weight of a single NC divided by the magnetic dipole moment of one dipole, $m$. The values of magnetization saturation observed for our CdSe NCs are within the range of values $(0.2-20 \mathrm{memu} / \mathrm{g})$ reported previously for CdSe NCs. ${ }^{46-49,61}$ The highest values of coercivity observed for our CdSe NCs $(200 \mathrm{mT})$ are above the range of values $(5-25 \mathrm{mT})$ reported for other CdSe $\mathrm{NCs},{ }^{46-49,61}$ but are comparable with the values reported for NCs of other diamagnetic materials. ${ }^{51,62,63}$

There are diverging reports concerning the magnetic properties of CdSe NCs, with some works reporting the observation of ferromagnetism (e.g., ref 46) and other works reporting the observation of paramagnetism (e.g., ref 50). In the specific case of our CdSe NCs, we observe magnetization loops revealing ferromagnetic behavior, although some of them display smaller coercivity. This shows that samples made of similar NCs can indeed exhibit different behavior. This is in line with the fact that different works, which use CdSe NCs produced in different ways and therefore with differences in terms of physical properties, report different magnetic behavior and indicates that the magnetic properties of CdSe NCs can be sample-dependent. Our goal is not to report the observation of ferromagnetism in CdSe NCs, neither to suggest that CdSe NCs are always ferromagnetic. It is also beyond the scope of our work to explain the origin of the apparent conflicting results published in the literature concerning the magnetic behavior of CdSe NCs. It is possible that both observations are correct, with the appearance of ferromagnetism or paramagnetism being dependent on particular characteristics of the specific CdSe NCs studied in different works, such as surface passivation, geometry, and chemistry. For example, in the case of $\mathrm{Au}$ nanoparticles, it has been found that the magnetic properties are strongly dependent on the type of capping agent. $^{64,65}$

Here, we instead focus on the description of the shape and amplitude of the observed angular-dependent magnetic resonance. As shall be explained below, this is done using a simple model based on magnetic dipole-dipole interactions between dipoles at the NCs surface. The possible origin of these surface spins will also be briefly discussed below. Importantly, we should note that our model does not require that the system of CdSe NCs is ferromagnetic and can be applied to both ferromagnetic and superparamagnetic systems. We now describe the theoretical framework to explain the angular dependence of our EMR spectra.

In a system consisting of noninteracting magnetic dipoles, each of the dipoles acts independently and the system displays a paramagnetic behavior, which in a powder sample should not show any magnetic anisotropy. ${ }^{60}$ However, if the magnetic dipoles interact via magnetic dipole-dipole interactions, ${ }^{15,66-69}$ the situation can be rather different. Assuming a system with $n_{\text {dip }}$ magnetic dipoles with a magnetic dipole moment $m$, which are distributed in a volume $V$, the magnetic free-energy density may be given by

$$
F=\mathbf{M} \cdot \mathbf{H}+F_{\text {dip }}
$$

where $\mathbf{M}$ is the total magnetization vector. From the magnetization loop measurements, as well as from measurements reported in refs $46,49,61$, we can infer that at the observed resonant magnetic fields $\left(H_{\text {res }} \geq 100 \mathrm{mT}\right)$, the magnetization of our NC ensembles is at (or close to) saturation, meaning that the magnetic dipoles are mutually aligned. This magnetic ordering is of course due to exchange coupling between the dipoles. We consider that the exchange interaction between dipoles is isotropic. Within this approach, the exchange coupling contributes to the free energy, $F$ as a constant that is independent of the direction of the magnetic dipoles, that is, does not depend on the direction of $\mathbf{H}$, and therefore does not contribute to the anisotropy of $F$. Typically, the symmetric and antisymmetric anisotropic terms of the exchange coupling are, respectively, 1 and 2 orders of magnitude smaller than the isotropic component of the exchange coupling. ${ }^{70,71} \mathrm{We}$ follow also previous studies that consider that the main contribution to the anisotropy observed in magnetic resonance spectra of nanoparticle systems results from the anisotropic dipole-dipole interaction. ${ }^{34,35,72}$ We should also note at this point that before each magnetic resonance measurement is started, the sample is always subjected to a magnetic field above $1000 \mathrm{mT}$, where it is magnetized. This results directly from the way the angular dependence of the EMR spectrum is obtained, where the measurement of each spectrum is always preceded by the measurement of another EMR spectrum where the magnetic field is scanned between 0 and $1000 \mathrm{mT}$. Thus, we consider that for all resonant fields, the magnetic dipoles responsible for the anisotropic magnetic resonance signal are collinear and so $\mathbf{M}$ has a magnitude, $M=n_{\text {dip }} m / V$. The first term in eq 1 corresponds to the Zeeman interaction and the second term accounts for the magnetic dipole-dipole interactions between the dipoles, which is given by

$$
\begin{aligned}
F_{\text {dip }} & =\frac{\mu_{0}}{V} \sum_{i} \sum_{j \neq i} \frac{m^{2}}{4 \pi r_{i j}{ }^{3}} \\
& \times\left\{1-3\left[\sin \alpha_{i j} \cos \left(\beta_{i j}-\alpha_{\mathrm{M}}\right) \sin \beta_{\mathrm{M}}-\cos \alpha_{i j} \cos \beta_{\mathrm{M}}\right]^{2}\right\}
\end{aligned}
$$

Here, $\mu_{0}$ is the vacuum permeability, $r_{i j}$ is the distance between magnetic dipoles $i$ and $j$, and the angles $\alpha_{i j}, \beta_{i j}, \alpha_{\mathrm{M}}$, and $\beta_{\mathrm{M}}$ are defined in Figure $3 a$. 

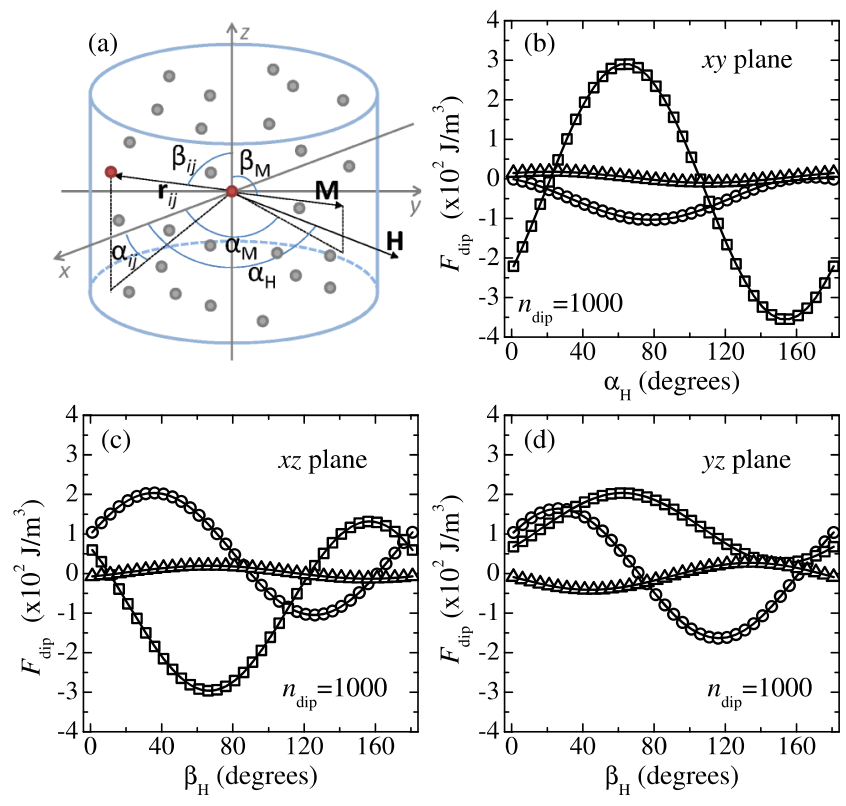

Figure 3. (a) Coordinate system used to describe $\mathbf{M}, \mathbf{H}$, and the vector, $\mathbf{r}_{i j}$ connecting magnetic dipoles $i$ and $j$. The cylinder and the grey spheres correspond to the sample volume and to magnetic dipoles randomly distributed in that volume, respectively. $(b-d)$ Energy, $F_{\text {dip }}$ calculated for three random arrangements of magnetic dipoles, with $n_{\text {dip }}=1000, m=1.73 \mu_{\mathrm{B}}$, and a density of 2 magnetic dipoles per $\mathrm{nm}^{3}$, as a function of orientation of $\mathbf{M}$ within the $x y$-, $x z$-, and $y z$-planes, respectively. The solid lines correspond to fittings using $F_{\text {eff }}$ given by eq 3 .

We consider a given number $n_{\text {dip }}$ of magnetic dipoles that are randomly distributed in a cylinder with a diameter equal to its height, corresponding to the geometry of our samples. Figure $3 \mathrm{~b}-\mathrm{d}$ shows the angular variation of $F_{\text {dip }}$ for rotation of $\mathbf{M}$ in the $x y$-, $x z$-, and $y z$-planes, respectively, obtained from three independent calculations, which correspond to three different computer-generated random arrangements of magnetic dipoles with the same $n_{\text {dip. }}$. The data shown in these figures is calculated for $n_{\text {dip }}=1000$, a density of magnetic dipoles of 2 dipoles per $\mathrm{nm}^{3}$, and $m=1.73 \mu_{\mathrm{B}}{ }^{a}$, where $\mu_{\mathrm{B}}$ is the Bohr magneton. From the data shown in Figure $3 b-d$, we observe that in all three calculations, $F_{\text {dip }}$ displays an angular variation in the three rotation planes. However, the angular variations are quantitatively different for each spatial arrangement of magnetic dipoles. Nonetheless, the calculated data already indicate that the term, $F_{\text {dip }}$ should result in an effective magnetic anisotropy in a random arrangement of magnetic dipoles. We have found that $F_{\text {dip }}$ can always be well parameterized by a simple energy density term of the form

$$
F_{\text {eff }}=\frac{\mu_{0}}{2} \mathbf{M} \cdot \mathbf{D} \cdot \mathbf{M}, \quad \mathbf{D}=\left[\begin{array}{lll}
D_{x x} & D_{x y} & D_{x z} \\
D_{y x} & D_{y y} & D_{y z} \\
D_{z x} & D_{z y} & D_{z z}
\end{array}\right]
$$

where $\mathbf{D}$ is a tensor that describes the effective dipolar interaction in the NC ensemble, with $D_{y x}=D_{x y}, D_{z x}=D_{x z}, D_{z y}$ $=D_{y z}$ and $D_{x x}+D_{y y}+D_{z z}=0$. As examples, the results of adjusting eq 3 to the angular variations of $F_{\text {dip }}$ given in Figure $3 \mathrm{~b}-\mathrm{d}$ are indicated as solid lines in the same figures. As can be seen, a rather good agreement is obtained. We would like to note that although the mathematical equation of our effective energy term, $F_{\text {eff }}$ is similar to that of the well-known magnetostatic energy comprising the so-called demagnetization tensor, $\mathbf{N})^{73}$ these two energy terms are not the same. Although the magnetostatic energy term typically describes the shape anisotropy of the magnetic material, which is not present in our system when the magnetic field is rotated in the $x y$ plane, our energy term, $F_{\text {eff }}$ describes the effective contribution of the magnetic dipole-dipole interactions between all dipoles to the energy of the NC ensemble. Thus, the characteristics of the tensor $\mathbf{D}$ in $F_{\text {eff }}$ and of the demagnetization tensor $\mathbf{N}$ are not necessarily the same. Indeed, we find that $\operatorname{tr}(\mathbf{D})$ is 0 , whereas the trace of the demagnetization tensor is known to be 1 .

Our analysis implies that the angular dependence of $H_{\text {res }}$ may be obtained from the resonance equation derived by Suhl and $\mathrm{Smit}^{74,75}$ in conjunction with the magnetic free energy given by eq 1 , with the quite complex dipolar energy term, $F_{\text {dip }}$ given simply by eq 3 . From this, a general equation for the angular variation of $H_{\text {res }}$, which depends on the elements of $\mathbf{D}$, can be obtained. This is given by eq S3 in the Supporting Information. For $\mathbf{H}$ oriented in the $x y$-plane, this general equation has a negligible dependence on $D_{x z}$ and $D_{y z}$ and, therefore, it can be approximated to

$$
\begin{aligned}
& \left(\frac{\omega}{\gamma}\right)^{2}-\frac{1}{2}\left\{2 C \sin \left(2 \alpha_{\mathrm{M}}\right)+B \cos \left(2 \alpha_{\mathrm{M}}\right)-H_{\text {res }} \cos \Delta\right\} \\
& \quad \times\left\{B \cos \left(2 \alpha_{\mathrm{M}}\right)+3 A+2\left[2 C \sin \left(2 \alpha_{\mathrm{M}}\right)+H_{\text {res }} \cos \Delta\right]\right\}=0
\end{aligned}
$$

where $\omega=2 \pi \nu$, with $\nu$ being the frequency of the applied microwave field, and $\gamma=2 \pi g \mu_{\mathrm{B}} / h$. Moreover, $h$ is the Planck constant, $\Delta=\alpha_{\mathrm{H}}-\alpha_{\mathrm{M}}$, and $g$ is the $g$-factor, which is set equal to 2. The dependence of $H_{\text {res }}$ on the orientation of the external magnetic field is determined by the parameters, $A=\mu_{0} M\left(D_{x x}+\right.$ $\left.D_{y y}\right), B=\mu_{0} M\left(D_{x x}-D_{y y}\right)$, and $C=\mu_{0} M D_{x y}$. The parameter $A$ gives the shift of the median of the angular variation of $H_{\text {res }}$ from $H=h \nu / g \mu_{\mathrm{B}}$. The amplitude of this angular variation depends only on $B$. The parameter $C$ sets the phase of the angular variation of $H_{\text {res }}$. The resonance condition in eq 4 is obtained for the equilibrium orientation of $\mathbf{M}$, for which $F$ is minimum $\left(\partial F / \partial \alpha_{M}=0\right),{ }^{73}$ which is given by

$$
2 C \cos \left(2 \alpha_{\mathrm{M}}\right)-B \sin \left(2 \alpha_{\mathrm{M}}\right)-2 H_{\text {res }} \sin \Delta=0
$$

Overlayered to the spectra shown in Figure 1, we plot the fitted angular variations of $H_{\text {res }}$ calculated from eqs 4 and 5 . As can be seen, the calculations describe quite well our experimental angular variations, $H_{\text {res }}$. These fits yield the values of $A=191.5 \mathrm{mT}$ and $B=204.6 \mathrm{mT}$ for sample $\mathrm{I}$, and $A$ $=122.6 \mathrm{mT}$ and $B=24.1 \mathrm{mT}$ for sample II. The values of $C$ obtained from these fits are irrelevant and set to $C=0 \mathrm{mT}$ because the phase of our experimental angular variations of $H_{\text {res }}$ is set arbitrarily, as mentioned above.

We have shown that the angular variation of the EMR spectra of our samples may be described by a model that considers magnetic dipoles interacting via magnetic dipoledipole interactions. These interactions may occur between magnetic dipoles at the surface of NCs, where unterminated sites may result in paramagnetic defects with a localized electron spin which has a magnetic dipole moment. ${ }^{43,76}$ In our NCs, most of the surface atoms should be passivated with organic ligands, but previous studies showed that only $\sim 70 \%$ of the atoms at the surface of CdSe NCs are passivated by ligands. ${ }^{77,78}$ Thus, in our CdSe NCs with $d_{\mathrm{NC}}=2.7 \mathrm{~nm}$, we 
expect that about 30 surface atoms remain unpassivated and result in paramagnetic defects that provide the magnetic dipoles in our samples. This amount is consistent with the value of 10-30 magnetic dipoles per CdSe NC estimated above from magnetization loop measurements.

To simulate the magnetic dipole-dipole interactions between surface magnetic dipoles in our samples, we generated ensembles of $n_{\mathrm{NC}}$ ligand-capped NCs with $d_{\mathrm{NC}}=2.7 \mathrm{~nm}$. For that, we distribute the NCs in a cylinder with a diameter equal to its height and a volume that corresponds to a volume fraction of NCs equal to 0.2. In random ensembles of NCs, the $\mathrm{NC}$ volume fraction is expected to be much smaller than that of ordered 3-dimensional arrays of NCs, such as an FCC NC superlattice, with a volume fraction of 0.74 . Each $\mathrm{NC}$ in the ensemble is placed with its ligand shell in contact with that of its neighbors. This situation is represented for two neighboring NCs in Figure 4a. The contact point between NCs is labeled as
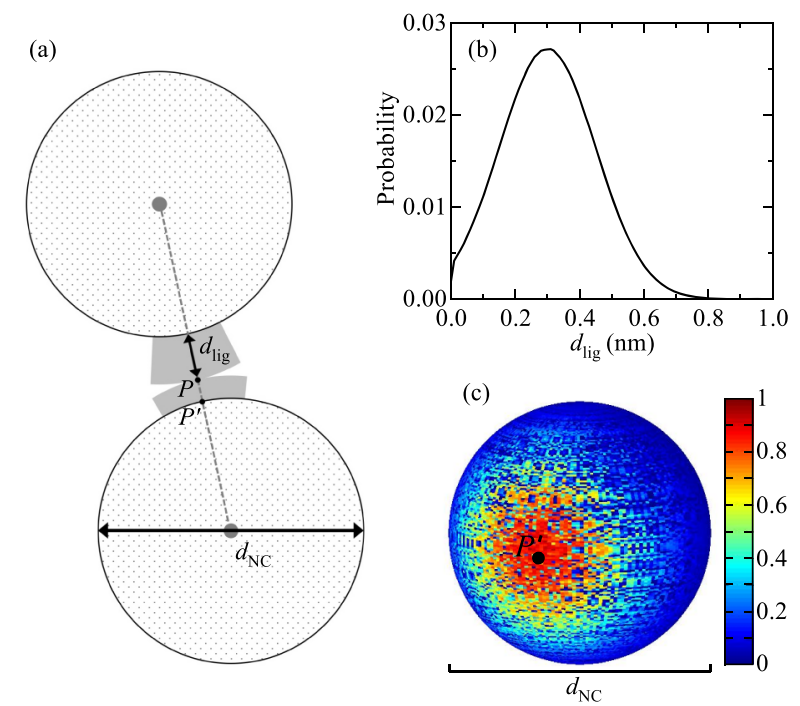

Figure 4. (a) Scheme of two neighboring ligand-capped NCs in contact with each other at the point $P$. The grey regions represent the thickness of the ligand shell of each NC at their mutual contact point, $P$. (b) Probability distribution of the ligand shell thickness, $d_{\text {lig. }}$ (c) Spatial probability distribution, $P\left(\mathbf{r}_{\text {dip }}\right)$ of the extra magnetic dipole that is placed at the NC surface when $d_{\mathrm{lig}}<0.3 \mathrm{~nm}$. The maximum of this distribution is located at the point $P^{\prime}$, which corresponds to the point on the NC surface that is closest to the contact point $P$.

$P$. As mentioned above, the ligand coverage of NCs is incomplete. Therefore, we define the thickness of each NC ligand shell at the NC's surface point that is nearest to $P$, which is indicated in one of the NCs in Figure 4a and labeled as $P^{\prime}$. For each $P^{\prime}$, the thickness of the NC ligand shell is picked randomly from the normal distribution shown in Figure $4 \mathrm{~b}$. We set the mean and width of this distribution equal to 0.3 $\mathrm{nm}$, in accordance with the previously reported calculations of the equilibrium separation between surfaces of ligand-capped NCs. ${ }^{79,80}$ On the surface of each NC in the ensemble, we randomly distribute 10 magnetic dipoles, separated from each other by at least the bond length of CdSe $(0.26 \mathrm{~nm})$. This is a conservative estimate for the amount of magnetic dipoles estimated as described above. It is also reasonable to assume that in regions of a NC surface where the ligand shell is thinner, which result from a lower coverage by ligands, the number of surface dipoles is larger. To account for this, we add one magnetic dipole on the NC surface when $d_{\text {lig }}<0.3 \mathrm{~nm}$.
The extra dipole is placed near the corresponding $P^{\prime}$, according to a Fisher distribution $P\left(\mathbf{r}_{\text {dip }}\right)=C(\kappa) \exp \left(\kappa \boldsymbol{\mu} \cdot \mathbf{r}_{\text {dip }}\right) .{ }^{81}$ Here, $C(\kappa)=\kappa / 4 \pi \sinh \kappa, \mathbf{r}_{\text {dip }}$ is the position of the magnetic dipole, and $\boldsymbol{\mu}$ and $\kappa$ determine the direction of the maximum and the width of $P\left(\mathbf{r}_{\text {dip }}\right)$, respectively. We set $P\left(\mathbf{r}_{\text {dip }}\right)$ with $\kappa=3$ and with maximum at the corresponding $P^{\prime}$. This value was chosen so that the extra dipole, resulting from incomplete passivation (thinner ligand shell), is located in the vicinity of the contact region between the NCs. The used $P\left(\mathbf{r}_{\text {dip }}\right)$ distribution is shown in Figure 4c.

We have calculated the distributions of the parameters $A$ and $B$ for different situations of $n_{\mathrm{NC}}$. For each situation, we have generated 2000 different ensembles of NCs, we have calculated $F_{\text {dip }}$ for each ensemble, and afterward, we have calculated the elements of the tensor $\mathbf{D}$ for each ensemble by adjusting eq 3 to $F_{\text {dip }}$. From these elements, we then calculated the parameters $A$ and $B$ for each ensemble. The distributions of these parameters are shown in Figure 5 for sets of 2000
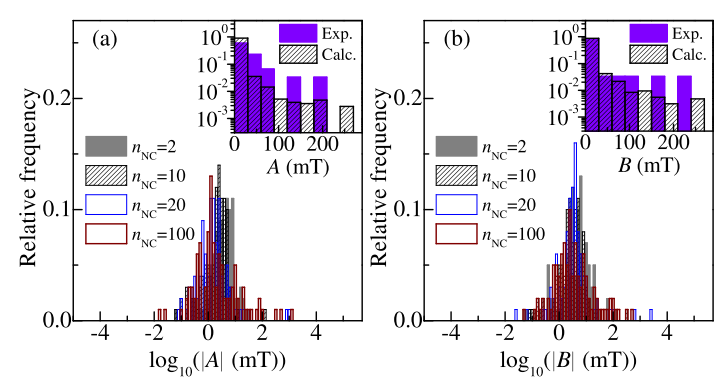

Figure 5. Distributions of the parameters (a) $A$ and (b) $B$ calculated for 2000 different computer-generated NC ensembles with $n_{\mathrm{NC}}=2$, 10,20 , and 100 . The insets compare the distributions of experimental values of $A$ and $B$ obtained from the EMR spectra with those calculated for NC ensembles with $n_{\mathrm{NC}}=100$.

ensembles of NCs with $n_{\mathrm{NC}}=2,10,20$, and 100 . Here, we see that the distributions of $A$ and $B$ obtained from our calculations span about 4 orders of magnitude and are not significantly dependent on $n_{\mathrm{NC}}$. We note that the experimental values of $A$ and $B$ obtained from the fits shown in Figure 1 ( $A$ $=191.5 \mathrm{mT}$ and $B=206.6 \mathrm{mT}$ for sample $\mathrm{I}$, and $A=122.6 \mathrm{mT}$ and $B=24.1 \mathrm{mT}$ for sample II) are within the ranges of values of $A$ and $B$ obtained from our calculations, for all $n_{\mathrm{NC}}$ considered. The insets of Figure 5a,b show the distributions of experimental values of $A$ and $B$ obtained from the EMR spectra of 30 different samples (purple bars). From comparison with the corresponding calculated distributions (black stripped bars), we see that our calculations are in good agreement with the wide distributions of values of $A$ and $B$ observed experimentally.

The calculations described above enable us also to further discuss the microscopic origin of the anisotropy of the energy term $F_{\text {dip }}$, and the consequent anisotropy of the EMR for random ensembles of NCs. Here, we should bear in mind that the dipole-dipole interaction is intrinsically anisotropic because its strength is different when the two (mutuallyaligned) magnetic moments of the interacting dipoles are aligned along the line connecting them compared to when they are aligned perpendicularly to this line. Hence, for a simple system with only two mutually aligned magnetic moments, $F_{\text {dip }}$ is always anisotropic. For a system with many randomly distributed magnetic dipoles, the energy term $F_{\text {dip }}$ in eq 2 accounts for all dipole-dipole interactions resulting from all 
magnetic dipole pairs present in the system, that is corresponds to the summation of all interactions between all magnetic dipole pairs. According to our calculations, for any given arrangement of randomly distributed magnetic dipoles in the $\mathrm{NC}$ ensemble, there are always a few magnetic dipole pairs that contribute much more to $F_{\text {dip }}$ than all other pairs. In other words, $F_{\text {dip }}$ is dominated by only a few dipole pairs, which are the pairs where the two magnetic dipoles are more closely spaced. Thus, even for a system with a large number of magnetic dipoles, like in our NC ensembles, $F_{\text {dip }}$ is dominated by only a few magnetic dipoles and the geometric arrangement of these few magnetic dipoles is effectively always anisotropic. From our calculations for magnetic dipoles randomly distributed on the NCs surface, $F_{\text {dip }}$ is dominated by only 2-4 dipole pairs. For most of the calculated NC ensembles, the 2-4 dipole pairs that dictate the anisotropy involve at least one dipole that is added as a result of a thinner surface shell, which is generated by the Fisher distribution as described above. Thus, our calculations suggest a link between incomplete (thinner) surface passivation and the appearance of surface dangling bonds (dipoles). Moreover, it becomes apparent that the dipole pairs contributing the most to $F_{\text {dip }}$ correspond to pairs where each dipole is located in different NCs and where the inter-dipole distance is in the range of a few angstroms. As $F_{\text {dip }}$ is always dominated by only a few dipole pairs, it is readily expected that different ensembles of statistically similar NCs, in terms of average number of surface dipoles and random distribution of these dipoles, may have very different configurations for the dominant dipole pairs, in terms of inter-dipole distances and orientations. Thus, we may understand why different ensembles of similar NCs, from the same synthesis batch or synthesized in a similar way, may exhibit very different values for the parameters $A$ and $B$.

\section{CONCLUSIONS}

In summary, we report the observation of magnetic anisotropy in ensembles of NCs randomly stacked in an isotropic shape using angular-dependent magnetic resonance. We show that the shape of the magnetic resonance angular dependence can be well described by a simple model that considers magnetic dipole-dipole interactions between dipoles randomly distributed in the NCs, possibly originating from incomplete NC surface passivation. The magnetic anisotropy results from the fact that the energy term arising from the magnetic dipoledipole interactions between all magnetic moments in the system is dominated by only a few dipole pairs, which always have an anisotropic geometric arrangement. As incomplete NC surface passivation is usually unavoidable, our study shows that magnetic anisotropy may be a general feature of NC systems.

\section{EXPERIMENTAL SECTION}

In this study, we used CdSe NCs with a diameter of $d_{\mathrm{NC}}=2.7$ $\mathrm{nm}$ produced via colloidal synthesis, ${ }^{82}$ capped with tri- $n$ octylphosphine (TOP), tri- $n$-octylphosphine oxide (TOPO), hexadecylamine (HDA), and dodecylphosphonic acid (DDPA). After synthesis, the NCs were washed to clean unreacted reagents from the dispersion. In this procedure, the NCs are isolated by precipitation with ethanol and redispersed in hexane or toluene. ${ }^{82,83}$ Details of the synthesis of the CdSe NCs used in this study are provided in the Supporting Information. To quantify the concentration of transition-metal contaminants, we performed elemental analyses by inductively coupled plasma-optical emission spectroscopy. The concentration of $\mathrm{Fe}$ impurities in our samples is about $1-2 \mathrm{ppb}$ (i.e., roughly about $1 \mathrm{Fe}$ atom per $2500 \mathrm{NCs}$ ), and the concentration of $\mathrm{Co}$ and $\mathrm{Ni}$ is below the detection limit of the technique $(<0.1 \mathrm{ppb})$.

EMR was performed with a few milligrams of dry NC powder filled into suprasil quartz tubes sealed under an inert atmosphere of $\mathrm{N}_{2}$. The sample volume corresponds to a cylinder with height approximately equal to the tube diameter. For our measurements, we used a Bruker ESP $300 \mathrm{E}$ continuous-wave X-band spectrometer (microwave frequency of $9.45 \mathrm{GHz}$ ) driven in the absorption mode. Magnetization loops were measured at room temperature in the field range from -1 to $1 \mathrm{~T}$ using a superconducting quantum interference device (SQUID) magnetometer Quantum Design MPMS-5S. Quartz tubes filled with NCs were placed in the center of long plastic tubes. The magnetic field was applied along the sample-tube axis.

\section{ASSOCIATED CONTENT}

\section{Supporting Information}

The Supporting Information is available free of charge at https://pubs.acs.org/doi/10.1021/acsomega.0c00279.

Synthesis protocol for the CdSe NCs used in this work, structural characterization of the CdSe NCs, comprising absorption spectrum, XRD pattern, TEM images of the CdSe NCs, and general equation for the angular variation of the EMR resonance magnetic field (PDF)

\section{AUTHOR INFORMATION}

\section{Corresponding Author}

Rui N. Pereira - i3N-Institute for Nanostructures, Nanomodelling and Nanofabrication, Department of Physics, University of Aveiro, 3810-193 Aveiro, Portugal; 10 orcid.org/ 0000-0003-1146-7506; Email: rnpereira@ua.pt

\section{Authors}

António J. S. Almeida - i3N-Institute for Nanostructures, Nanomodelling and Nanofabrication, Department of Physics, University of Aveiro, 3810-193 Aveiro, Portugal; NanoElectronics Group, MESA+ Institute for Nanotechnology, University of Twente, 7522 NB Enschede, The Netherlands

Ayaskanta Sahu - Optical Materials Engineering Laboratory, ETH Zurich, 8092 Zurich, Switzerland; Department of Chemical and Biomolecular Engineering, New York University, Brooklyn 11201, New York, United States; 이이.org/00000002-1508-0213

David J. Norris - Optical Materials Engineering Laboratory, ETH Zurich, 8092 Zurich, Switzerland; 이잉.org/00000002-3765-0678

Gleb N. Kakazei - Departamento de Física e Astronomia, IFIMUP and IN-Institute of Nanoscience and Nanotechnology, Universidade do Porto, 4169-007 Porto, Portugal

Haripriya Kannan - Department of Chemical and Biomolecular Engineering, New York University, Brooklyn 11201, New York, United States

Martin S. Brandt - Walter Schottky Institut and PhysikDepartment, Technische Universität München, 85748 Garching, Germany

Martin Stutzmann - Walter Schottky Institut and PhysikDepartment, Technische Universität München, 85748 Garching, Germany 
Complete contact information is available at:

https://pubs.acs.org/10.1021/acsomega.0c00279

\section{Notes}

The authors declare no competing financial interest.

\section{ACKNOWLEDGMENTS}

This work has been funded by FCT via projects no. PTDC/ 280/FIS/112885/2009, no. RECI/FIS-NAN/0183/2012, no. UID/CTM/50025/2019, no. NORTE-01-0145-FEDER022096, and no. MIT-EXPL/IRA/0012/2017 and Grant SFRH/BD/79223/2011, by the Swiss National Science Foundation under award no. 200021-140617, and by Fundação Calouste Gulbenkian via Prémio de Estímulo à Investigação. This work has been also developed in the scope of the Project i3N (project no. UID/CTM/50025/2019), financed by national funds through the Fundação para a Ciência e a Tecnologia/Ministério da Educação e Ciências (FCT/MEC) and when applicable cofinanced by FEDER under the PT2020 Partnership Agreement, and the Project i3N, UIDB/50025/2020 and UIDP/50025/2020, financed by national funds through the FCT/MEC. G.N.K. acknowledges the Network of Extreme Conditions Laboratories-NECL. H.K. has been (partially) supported by the Schlumberger Foundation Faculty for the Future Program. We gratefully acknowledge support for instrument use, scientific and technical assistance from the Imaging Facility of CUNY Advanced Science Research Center, NYU shared Instrumentation Facility through the Materials Research Science and Engineering Center (MRSEC) and MRI programs of the National Science Foundation under award numbers DMR1420073 and DMR-0923251. We are grateful for the assistance of Tony $\mathrm{Hu}$ at the Department of Chemistry of New York University with the X-ray analysis, and we thank the support to the X-ray facility by the National Science Foundation under Award Numbers CRIF/CHE-0840277 and by the NSF MRSEC Program under award numbers DMR-0820341 and DMR-1420073.

\section{ADDITIONAL NOTE}

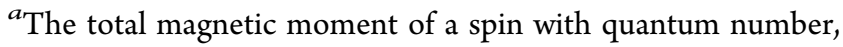
$S$ is given by $m=\sqrt{S(S+1)} \mu_{\mathrm{B}}$. Thus, for a spin with $S=1 / 2$, we have $m=1.73 \mu_{\mathrm{B}}$.

\section{REFERENCES}

(1) Alivisatos, A. P. Semiconductor Clusters, Nanocrystals, and Quantum Dots. Science 1996, 271, 933.

(2) Murray, C. B.; Norris, D. J.; Bawendi, M. G. Synthesis and characterization of nearly monodisperse $\mathrm{CdE}(\mathrm{E}=$ sulfur, selenium, tellurium) semiconductor nanocrystallites. J. Am. Chem. Soc. 1993, $115,8706$.

(3) Gupta, A.; Swihart, M. T.; Wiggers, H. Luminescent Colloidal Dispersion of Silicon Quantum Dots from Microwave Plasma Synthesis: Exploring the Photoluminescence Behavior Across the Visible Spectrum. Adv. Funct. Mater. 2009, 19, 696.

(4) Mangolini, L.; Thimsen, E.; Kortshagen, U. High-Yield Plasma Synthesis of Luminescent Silicon Nanocrystals. Nano Lett. 2005, 5, 655.

(5) Manna, L.; Milliron, D. J.; Meisel, A.; Scher, E. C.; Alivisatos, A. P. Controlled growth of tetrapod-branched inorganic nanocrystals. Nat. Mater. 2003, 2, 382.

(6) Murray, C. B.; Kagan, C. R.; Bawendi, M. G. Synthesis and Characterization of Monodisperse Nanocrystals and Close-Packed Nanocrystal Assemblies. Annu. Rev. Mater. Sci. 2000, 30, 545.
(7) Talapin, D. V.; Lee, J.-S.; Kovalenko, M. V.; Shevchenko, E. V. Prospects of Colloidal Nanocrystals for Electronic and Optoelectronic Applications. Chem. Rev. 2010, 110, 389.

(8) Majetich, S. A.; Jin, Y. Magnetization Directions of Individual Nanoparticles. Science 1999, 284, 470.

(9) Stamm, C.; Marty, F.; Vaterlaus, A.; Weich, V.; Egger, S.; Maier, U.; Ramsperger, U.; Fuhrmann, H.; Pescia, D. Two-Dimensional Magnetic Particles. Science 1998, 282, 449.

(10) Lederman, M.; Schultz, S.; Ozaki, M. Measurement of the Dynamics of the Magnetization Reversal in Individual Single-Domain Ferromagnetic Particles. Phys. Rev. Lett. 1994, 73, 1986.

(11) Wernsdorfer, W.; Orozco, E. B.; Hasselbach, K.; Benoit, A.; Barbara, B.; Demoncy, N.; Loiseau, A.; Pascard, H.; Mailly, D. Experimental Evidence of the Néel-Brown Model of Magnetization Reversal. Phys. Rev. Lett. 1997, 78, 1791.

(12) Puntes, V. F.; Krishnan, K. M.; Alivisatos, A. P. Colloidal nanocrystal shape and size control: the case of cobalt. Science 2001, 291, 2115.

(13) Skumryev, V.; Stoyanov, S.; Zhang, Y.; Hadjipanayis, G.; Givord, D.; Nogués, J. Beating the superparamagnetic limit with exchange bias. Nature 2003, 423, 850.

(14) Sahoo, S.; Petracic, O.; Kleemann, W.; Stappert, S.; Dumpich, G.; Nordblad, P.; Cardoso, S.; Freitas, P. P. Cooperative versus superparamagnetic behavior of dense magnetic nanoparticles in $\mathrm{Co}_{80} \mathrm{Fe}_{20} / \mathrm{Al}_{2} \mathrm{O}_{3}$ multilayers. Appl. Phys. Lett. 2003, 82, 4116.

(15) Puntes, V. F.; Gorostiza, P.; Aruguete, D. M.; Bastus, N. G.; Alivisatos, A. P. Collective behaviour in two-dimensional cobalt nanoparticle assemblies observed by magnetic force microscopy. Nat. Mater. 2004, 3, 263.

(16) Chen, J.; Dong, A.; Cai, J.; Ye, X.; Kang, Y.; Kikkawa, J. M.; Murray, C. B. Collective dipolar interactions in self-assembled magnetic binary nanocrystal superlattice membranes. Nano Lett. 2010, 10, 5103.

(17) Todorovic, M.; Schultz, S.; Wong, J.; Scherer, A. Writing and reading of single magnetic domain per bit perpendicular patterned media. Appl. Phys. Lett. 1999, 74, 2516.

(18) Sun, S.; Murray, C. B.; Weller, D.; Folks, L.; Moser, A. Monodispersse FePt Nanoparticles and Ferromagnetic FePt Nanocrystal Superlattices. Science 2000, 287, 1989.

(19) Le Gall, C.; Besombes, L.; Boukari, H.; Kolodka, R.; Cibert, J.; Mariette, H. Optical Spin Orientation of a Single Manganese Atom in a Semiconductor Quantum Dot Using Quasiresonant Photoexcitation. Phys. Rev. Lett. 2009, 102, 127402.

(20) Cowburn, R. P.; Welland, M. E. Room temperature magnetic quantum cellular automata. Science 2000, 287, 1466.

(21) Zabow, G.; Dodd, S.; Moreland, J.; Koretsky, A. Microengineered local field control for high-sensitivity multispectral MRI. Nature 2008, 453, 1058.

(22) Derfus, A. M.; von Maltzahn, G.; Harris, T. J.; Duza, T.; Vecchio, K. S.; Ruoslahti, E.; Bhatia, S. N. Remotely Triggered Release from Magnetic Nanoparticles. Adv. Mater. 2007, 19, 3932.

(23) Lee, J.-H.; Jang, J.-t.; Choi, J.-s.; Moon, S. H.; Noh, S.-h.; Kim, J.-w.; Kim, J.-G.; Kim, I.-S.; Park, K. I.; Cheon, J. Exchange-coupled magnetic nanoparticles for efficient heat induction. Nat. Nanotechnol. $2011,6,418$.

(24) Fortin, J.-P.; Wilhelm, C.; Servais, J.; Ménager, C.; Bacri, J.-C.; Gazeau, F. Size-Sorted Anionic Iron Oxide Nanomagnets as Colloidal Mediators for Magnetic Hyperthermia. J. Am. Chem. Soc. 2007, 129, 2628.

(25) Thomas, C. R.; Ferris, D. P.; Lee, J.-H.; Choi, E.; Cho, M. H.; Kim, E. S.; Stoddart, J. F.; Shin, J.-S.; Cheon, J.; Zink, J. I. Noninvasive Remote-Controlled Release of Drug Molecules in Vitro Using Magnetic Actuation of Mechanized Nanoparticles. J. Am. Chem. Soc. 2010, 132, 10623.

(26) Liu, T.-Y.; Hu, S.-H.; Liu, D.-M.; Chen, S.-Y.; Chen, I.-W. Biomedical nanoparticle carriers with combined thermal and magnetic responses. Nano Today 2009, 4, 52.

(27) Thiesen, B.; Jordan, A. Clinical applications of magnetic nanoparticles for hyperthermia. Int. J. Hyperthermia 2008, 24, 467. 
(28) Levy, A.; Dayan, A.; Ben-David, M.; Gannot, I. A new thermography-based approach to early detection of cancer utilizing magnetic nanoparticles theory simulation and in vitro validation. Nanomedicine 2010, 6, 786.

(29) Huang, H.; Delikanli, S.; Zeng, H.; Ferkey, D. M.; Pralle, A. Remote control of ion channels and neurons through magnetic-field heating of nanoparticles. Nat. Nanotechnol. 2010, 5, 602.

(30) Xiong, Z.; Cao, L. High magnetic-dielectric tunability in $\mathrm{Ni}$ nanocrystals embedded in $\mathrm{BaTiO}_{3}$ films. J. Alloys Compd. 2019, 785, 200.

(31) Zhou, Z.; Li, J.; Xiong, Z.; Cao, L.; Fu, Y.; Gao, Z. Reducing transition temperature and diluting brown-yellow color of $\mathrm{VO}_{2}$ films via embedding Ag particles periodic arrays. Sol. Energy Mater. Sol. Cells 2020, 206, 110303.

(32) Shi, D.; Xiong, Z.; Li, J.; Luo, B. Q.; Fang, L. M.; Xia, Y. H.; Gao, Z. Electron transition and electron-hole recombination processes in epitaial $\mathrm{BaTiO}_{3}$ films with embedded Co nanocrystals. Mater. Res. Express 2019, 6, 105021.

(33) Xu, J.; Wang, L.; Sun, Y.; Zhang, J.; Zhang, C.; Zhang, M. Fabrication of porous $\mathrm{MgCo}_{2} \mathrm{O}_{4}$ nanoneedle arrays/Ni foam as an advanced electrode material for asymmetric supercapacitors. J. Alloys Compd. 2019, 779, 100.

(34) Tomita, S.; Akamatsu, K.; Shinkai, H.; Ikeda, S.; Nawafune, H.; Mitsumata, C.; Kashiwagi, T.; Hagiwara, M. Tuning magnetic interactions in ferromagnetic-metal nanoparticle systems. Phys. Rev. B: Condens. Matter Mater. Phys. 2005, 71, No. 180414(R).

(35) Schmool, D. S.; Rocha, R.; Sousa, J. B.; Santos, J. A. M.; Kakazei, G. N.; Garitaonandia, J. S.; Lezama, L. The role of dipolar interactions in magnetic nanoparticles: Ferromagnetic resonance in discontinuous multilayers. J. Appl. Phys. 2007, 101, 103907.

(36) Butera, A.; Zhou, J. N.; Barnard, J. A. Ferromagnetic resonance in as-deposited and annealed $\mathrm{Fe}-\mathrm{SiO}_{2}$ heterogeneous thin films. Phys. Rev. B: Condens. Matter Mater. Phys. 1999, 60, 12270.

(37) Sugawara, A.; Scheinfein, M. R. Room-temperature dipole ferromagnetism in linear-self-assembling mesoscopic $\mathrm{Fe}$ particle arrays. Phys. Rev. B: Condens. Matter Mater. Phys. 1997, 56, R8499.

(38) Noginova, N.; Barnakov, Y.; Radocea, A.; Atsarkin, V. A. Role of dipole-dipole interactions in half-field quantum transitions in magnetic nanoparticles. J. Magn. Magn. Mater. 2011, 323, 2264.

(39) Varón, M.; Beleggia, M.; Kasama, T.; Harrison, R. J.; DuninBorkowski, R. E.; Puntes, V. F.; Frandsen, C. Dipolar Magnetism in Ordered and Disordered Low-Dimensional Nanoparticle Assemblies. Nat. Sci. Rep. 2013, 3, 1234.

(40) Kakazei, G. N.; Kravetz, A. F.; Lesnik, N. A.; Pereira de Azevedo, M. M.; Bondarkova, Y. G.; Silantiev, V. I.; Sousa, J. B. Influence of Co-evaporation Technique on the Structural and Magnetic Properties of $\mathrm{CoCu}$ Granular Films. J. Magn. Magn. Mater. 1999, 196-197, 29.

(41) Dubowik, J. Shape anisotropy of magnetic heterostructures. Phys. Rev. B: Condens. Matter Mater. Phys. 1996, 54, 1088. Kakazei, G. N.; Kravets, A. F.; Lesnik, N. A.; Pereira de Azevedo, M. M.; Pogorelov, Y. G.; Sousa, J. B. Ferromagnetic resonance in granular thin films. J. Appl. Phys. 1999, 85, 5654.

(42) Iwata, T. A Diagonal Sum Rule Concerning Demagnetization Tensors in Composite Bodies. J. Appl. Phys. 1968, 39, 3094.

(43) Rodina, A.; Efros, A. L. Magnetic Properties of Nonmagnetic Nanostructures: Dangling Bond Magnetic Polaron in CdSe Nanocrystals. Nano Lett. 2015, 15, 4214.

(44) Biadala, L.; Shornikova, E. V.; Rodina, A. V.; Yakovlev, D. R.; Siebers, B.; Aubert, T.; Nasilowski, M.; Hens, Z.; Dubertret, B.; Efros, A. L.; Bayer, M. Magnetic polaron on dangling-bond spins in CdSe colloidal nanocrystals. Nat. Nanotechnol. 2017, 12, 569.

(45) Almeida, A. J.; Sahu, A.; Riedinger, A.; Norris, D. J.; Brandt, M. S.; Stutzmann, M.; Pereira, R. N. Charge Trapping Defects in CdSe Nanocrystal Quantum Dots. J. Phys. Chem. C 2016, 120, 13763.

(46) Seehra, M. S.; Dutta, P.; Neeleshwar, S.; Chen, Y.-Y.; Chen, C. L.; Chou, S. W.; Chen, C. C.; Dong, C.-L.; Chang, C.-L. SizeControlled Ex-nihilo Ferromagnetism in Capped CdSe Quantum Dots. Adv. Mater. 2008, 20, 1656.
(47) Singh, S. B.; Limaye, M. V.; Date, S. K.; Kulkarni, S. K. Room temperature ferromagnetism in thiol-capped $\mathrm{CdSe}$ and $\mathrm{CdSe}: \mathrm{Cu}$ nanoparticles. Chem. Phys. Lett. 2008, 464, 208.

(48) Singh, B. S.; Limaye, M. V.; Date, S. K.; Gokhale, S.; Kulkarni, $\mathrm{S}$. K. Iron substitution in CdSe nanoparticles: Magnetic and optical properties. Phys. Rev. B: Condens. Matter Mater. Phys. 2009, 80, 235421.

(49) Singh, S.; Rath, M. C.; Singh, A. K.; Mukherjee, T.; Jayakumar, O. D.; Tyagi, A. K.; Sarkar, S. K. CdSe Nanoparticles Grown via Radiolytic Methods in Aqueous Solutions. Rad. Phys. Chem. 2011, 80, 736 .

(50) Meulenberg, R. W.; Lee, J. R. I.; McCall, S. K.; Hanif, K. M.; Haskel, D.; Lang, J. C.; Terminello, L. J.; van Buuren, T. Evidence for Ligand-Induced Paramagnetism in CdSe Quantum Dots. J. Am. Chem. Soc. 2009, 131, 6888.

(51) Crespo, P.; Litran, R.; Rojas, T. C.; Multigner, M.; de la Fuente, J. M.; Sanchez-Lopez, J. C.; Garcia, M. A.; Hernando, A.; Penades, S.; Fernandez, A. Permanent Magnetism, Magnetic Anisotropy, and Hysteresis of Thiol-Capped Gold Nanoparticles. Phys. Rev. Lett. 2004, 93, 087204.

(52) Garitaonandia, J. S.; Insausti, M.; Goikolea, E.; Suzuki, M.; Cashion, J. D.; Kawamura, N.; Ohsawa, H.; Gil de Muro, I.; Suzuki, K.; Plazaola, F.; Rojo, T. Chemically Induced Permanent Magnetism in $\mathrm{Au}, \mathrm{Ag}$, and $\mathrm{Cu}$ Nanoparticles: Localization of the Magnetism by Element Selective Techniques. Nano Lett. 2008, 8, 661.

(53) Dehn, M. H.; Arseneau, D. J.; Buck, T.; Cortie, D. L.; Fleming, D. G.; King, S. R.; MacFarlane, W. A.; McDonagh, A. M.; McFadden, R. M. L.; Mitchell, D. R. G.; Kiefl, R. F. Nature of magnetism in thiolcapped gold nanoparticles investigated with Muon spin rotation. Appl. Phys. Lett. 2018, 112, 053105.

(54) Agrachev, M.; Antonello, S.; Dainese, T.; Ruzzi, M.; Zoleo, A.; Aprà, E.; Govind, N.; Fortunelli, A.; Sementa, L.; Maran, F. Magnetic Ordering in Gold Nanoclusters. ACS Omega 2017, 2, 2607.

(55) Tuboltsev, V.; Savin, A.; Pirojenko, A.; Räisänen, J. Magnetism in Nanocrystalline Gold. ACS Nano 2013, 7, 6691.

(56) Viloria, M. G.; Weick, G.; Weinmann, D.; Jalabert, R. A. Orbital magnetism in ensembles of gold nanoparticles. Phys. Rev. B 2018, 98, 195417.

(57) Garcia, M. A.; Merino, J. M.; Fernández Pinel, E.; Quesada, A.; de la Venta, J.; Ruíz González, M. L.; Castro, G. R.; Crespo, P.; Llopis, J.; González-Calbet, J. M.; Hernando, A. Magnetic Properties of ZnO Nanoparticles. Nano Lett. 2007, 7, 1489.

(58) Peng, H.; Xiang, H. J.; Wei, S.-H.; Li, S.-S.; Xia, J.-B.; Li, J. Origin and Enhancement of Hole-Induced Ferromagnetism in FirstRow d $\mathrm{d}^{0}$ Semiconductors. Phys. Rev. Lett. 2009, 102, 017201.

(59) Chen, Y.-C.; Wang, Z.; Leineweber, A.; Baier, J.; Tietze, T.; Phillipp, F.; Schütz, G.; Goering, E. Effect of surface configurations on the room-temperature magnetism of pure $\mathrm{ZnO}$. J. Mater. Chem. C 2016, 4, 4166.

(60) Weil, J. A.; Bolton, J. R.; Wertz, J. E. Electron Paramagnetic Resonance: Elementary Theory and Practical Applications; WileyInterscience: New York, USA, 1994.

(61) Kumar, S.; Kumar, S.; Verma, N. K.; Chakarvarti, S. K. Room temperature magnetism in $\mathrm{Ni}$-doped CdSe nanoparticles. J. Mater. Sci.: Mater. Electron. 2011, 22, 901.

(62) Muñoz-Márquez, M. A.; Guerrero, E.; Fernandez, A.; Fernández, P.; Hernando, A.; Lucena, R.; Conesa, J. C. Permanent magnetism in phosphine- and chlorine-capped gold: From clusters to nanoparticles. J. Nanopart. Res. 2010, 12, 1307.

(63) Sundaresan, A.; Bhargavi, R.; Rangarajan, N.; Siddesh, U.; Rao, C. N. R. Ferromagnetism as a universal feature of nanoparticles of the otherwise nonmagnetic oxides. Phys. Rev. B: Condens. Matter Mater. Phys. 2006, 74, No. 161306(R).

(64) Hori, H.; Yamamoto, Y.; Iwamoto, T.; Miura, T.; Teranishi, T.; Miyake, M. Diameter dependence of ferromagnetic spin moment in Au nanocrystals. Phys. Rev. B: Condens. Matter Mater. Phys. 2004, 69, 174411.

(65) de la Presa, P.; Multigner, M.; de la Venta, J.; García, M. A.; Ruiz-González, M. L. Structural and magnetic characterization of oleic 
acid and oleylamine-capped gold nanoparticles. J. Appl. Phys. 2006, $100,123915$.

(66) Russier, V.; Petit, C.; Legrand, J.; Pileni, M. P. Collective magnetic properties of cobalt nanocrystals self-assembled in a hexagonal network: Theoretical model supported by experiments. Phys. Rev. B: Condens. Matter Mater. Phys. 2000, 62, 3910.

(67) Fried, T.; Shemer, G.; Markovich, G. Ordered TwoDimensional Arrays of Ferrite Nanoparticles. Adv. Mater. 2001, 13, 1158.

(68) Song, Q.; Zhang, Z. J. Shape Control and Associated Magnetic Properties of Spinel Cobalt Ferrite Nanocrystals. J. Am. Chem. Soc. 2004, 126, 6164.

(69) Vestal, C. R.; Song, Q.; Zhang, Z. J. Effects of Interparticle Interactions upon the Magnetic Properties of $\mathrm{CoFe} 2 \mathrm{O} 4$ and MnFe2O4 Nanocrystals. J. Phys. Chem. B 2004, 108, 18222.

(70) Crépieux, A.; Lacroix, C. Dzyaloshinsky-Moriya Interactions Induced by Symmetry Breaking at a Surface. J. Magn. Magn. Mater. 1998, 182, 341-349.

(71) Moriya, T. Anisotropic Superexchange Interaction and Weak Ferromagnetism. Phys. Rev. 1960, 120, 91.

(72) Mitsumata, C.; Tomita, S.; Hagiwara, M.; Akamatsu, K. Electron Magnetic Resonance in Interacting Ferromagnetic-metal Nanoparticle Systems: Experiment and Numerical Simulation. J. Phys.: Condens. Matter 2010, 22, 016005.

(73) Vonsovskii, S. V. Ferromagnetic Resonance, 1st ed.; Pergamon Press, 1966.

(74) Suhl, H. Ferromagnetic Resonance in Nickel Ferrite Between One and Two Kilomegacycles. Phys. Rev. 1955, 97, 555.

(75) Smit, J.; Beljers, G. Ferromagnetic resonance absorption in $\mathrm{BaFe}_{2} \mathrm{O}_{1}$ 9, a highly anisotropic crystal. Philips Res. Rep. 1955, 10, 113.

(76) Neeleshwar, S.; Chen, C. L.; Tsai, C. B.; Chen, Y. Y.; Chen, C. C.; Shyu, S. G.; Seehra, M. S. Size-dependent properties of CdSe quantum dots. Phys. Rev. B: Condens. Matter Mater. Phys. 2005, 71, No. 201307(R).

(77) Katari, J. E. B.; Colvin, V. L.; Alivisatos, A. P. X-ray Photoelectron Spectroscopy of CdSe Nanocrystals with Applications to Studies of the Nanocrystal Surface. J. Phys. Chem. 1994, 98, 4109.

(78) Taylor, J.; Kippeny, T.; Rosenthal, S. J. Surface Stoichiometry of CdSe Nanocrystals Determined by Rutherford Backscattering Spectroscopy. J. Cluster Sci. 2001, 12, 571.

(79) Schapotschnikow, P.; Vlugt, T. J. H. Understanding Interactions between Capped Nanocrystals: Three-body and Chain Packing Effects. J. Chem. Phys. 2009, 131, 124705.

(80) Schapotschnikow, P.; Pool, R.; Vlugt, T. J. H. Molecular Simulations of Interacting Nanocrystals. Nano Lett. 2008, 8, 2930.

(81) Fisher, R. Dispersion on a Sphere. Proc. R. Soc. London, Ser. A 1953, 217, 295.

(82) Sahu, A.; Kang, M. S.; Kompch, A.; Notthoff, C.; Wills, A. W.; Deng, D.; Winterer, M.; Norris, D. J. Electronic Impurity Doping in CdSe Nanocrystals. Nano Lett. 2012, 12, 2587.

(83) Sahu, A.; Qi, L.; Kang, M. S.; Deng, D.; Norris, D. J. Facile Synthesis of Silver Chalcogenide $\left(\mathrm{Ag}_{2} \mathrm{E}, \mathrm{E}=\mathrm{Se}, \mathrm{S}, \mathrm{Te}\right)$ Semiconductor Nanocrystals. J. Am. Chem. Soc. 2011, 133, 6509. 